CASE REPORT

\title{
Biclonal Gammopathy of Iga Kappa Variant in a Patient with Systemic Lupus Erythematosus: A Case Report
}

\author{
${ }^{1}$ HAFIR Laboratory of Medical Analysis, Morocco \\ ${ }^{2}$ Medicine Service A, Regional Hospital of Beni Mellal, Morocco \\ ${ }^{3}$ Laboratory of Medical Analysis, Provincial Hospital of Fkih Ben Salah, Morocco \\ ${ }^{4}$ TOUZANI Laboratory of Medical Analysis, Boulevard of Moulay Ali Cherif, Morocco \\ ${ }^{5}$ Faculty of Sciences and Techniques, University My Slimane, Morocco
}

Hicham Esselmani ${ }^{1}$, Asmaa Yassine ${ }^{2}$, Boutaina Akhatar ${ }^{3}$, Otmane Touzani $^{4}$ and Abderrazak Lfakir ${ }^{5}$

*Corresponding author: Hicham Esselmani, HAFIR Laboratory of Medical Analysis, Lakouacem, street $28 n^{\circ} 12$. Fkih Ben Salah, Morocco

\begin{abstract}
The association of systemic lupus erythematosus and multiple myeloma is an uncommon event. Biclonal gammopathy is a rare case, which accounts for only $1.5 \%$ of all myelomas but biclonal gammopathy of IgA kappa variant is extremely rare. We report this unusual case in which the simultaneous diagnosis of biclonal gammopathy of IgA kappa variants and systemic lupus erythematosus was made.
\end{abstract}

\section{Keywords}

Systemic lupus erythematosus, Multiple myeloma, Biclonal gammopathy, IgA, Kappa chain

\section{Introduction}

It has been suggested that patients affected by autoimmune diseases are more prone to developing malignancies, and especially lymphoma [1]. Although the coexistence of multiple myeloma (MM) and systemic lupus erythematosus (SLE) has been described in humans, it remains extremely rare [1]. $\mathrm{MM}$ is characterized by the production of $\mathrm{M}$ protein. The monoclonal protein is detected by serum protein electrophoresis as a single discrete band (M-band) most often in the gamma globulin region. However, it may rarely show a simultaneous presence of two distinct $M-$ bands as seen in $1.5 \%$ of cases [2]. We report a case of simultaneous diagnosis of biclonal gammopathy with a rare combination of immunoglobulin (IgA/IgA Kappa) in a 56-year-old man and SLE.

\section{Case Report}

A 56-year-old man has a past medical history of renal failure (secondary to type 2 diabetes diagnosed 11 years before) manifesting fatigue, general weakness, undocumented weight loss, a photosensitive rash and intermittent arthralgias to the hospital. His temperature was $38.5^{\circ} \mathrm{C}$, heart rate was 72 beats/ $\mathrm{min}$, and blood pressure was $135 / 85 \mathrm{mmHg}$. Physical examination revealed persistent malar erythema and transient indurated erythema on the upper extremities and trunk. He had non-erosive arthritis on shoulders, elbows, and knees. He did not present with swollen fingers, alopecia, lymphadenopathy or oral ulcers.

Complete blood count, blood biochemical parameters and auto-immune serologic test results are detailed in Table 1. Serum electrophoresis on agarose gel (Sebia) revealed two sharp discrete bands. Band-1 $(10.21 \mathrm{~g} / \mathrm{l})$ is seen in the Beta 2 region and Band-2 $(3.03 \mathrm{~g} / \mathrm{l})$ is seen in the gamma region (Figure 1). Serum electrophoresis repeated after the serum pretreated with beta mercaptoethanol showed two distinct bands. On subjecting to the Immunofixation, it is found that both bands are of IgA-Kappa type (Figure 2 ). The urine was strongly positive (++) for protein. 
Table 1: Laboratory findings.

\begin{tabular}{|l|l|l|}
\hline Variable & Results & Reference values \\
\hline Red-cell count $\left({ }^{*} 10^{\wedge} 6 / \mu \mathrm{L}\right)$ & 4.37 & $4.1-6$ \\
\hline Hemoglobin $(\mathrm{g} / \mathrm{L})$ & 130 & $130-170$ \\
\hline Hematocrit $(\%)$ & 0.88 & $0.8-0.95$ \\
\hline White-cell count $\left({ }^{*} 10^{\wedge} 9 / \mathrm{L}\right)$ & 6.6 & $4.0-10$ \\
\hline Platelet count $\left({ }^{*} 10^{\wedge} / \mathrm{L}\right)$ & 126 & $150-450$ \\
\hline Erythrocyte sedimentation rate $(\mathrm{ESR})(\mathrm{mm} / \mathrm{h})$ & 93 & $<10$ \\
\hline Urea nitrogen $(\mathrm{mmol} / \mathrm{L})$ & 14 & $3.6-9.5$ \\
\hline Creatinine $(\mu \mathrm{mol} / \mathrm{L})$ & 318 & $60-130$ \\
\hline Proteinuria $(\mathrm{g} \mathrm{per} 24 \mathrm{~h})$ & 0.83 & $<0.15$ \\
\hline ANA & Positive $(1: 640)$ & Negative \\
\hline Anti-dsDNA $(\mathrm{AU} / \mathrm{ml})$ & 183 & $<25$ \\
\hline Anti-Sm $(\mathrm{U} / \mathrm{ml})$ & 1.19 & $<25$ \\
\hline Anti-SSA $(\mathrm{U} / \mathrm{ml})$ & 5.34 & $<25$ \\
\hline Anti-SSB $(\mathrm{U} / \mathrm{ml})$ & 2.9 & $<25$ \\
\hline Anti-Phospholipid $(\mathrm{U} / \mathrm{ml})$ & 1.43 & $<10$ \\
\hline
\end{tabular}

\section{(CAPILLARYS SEBIA)}

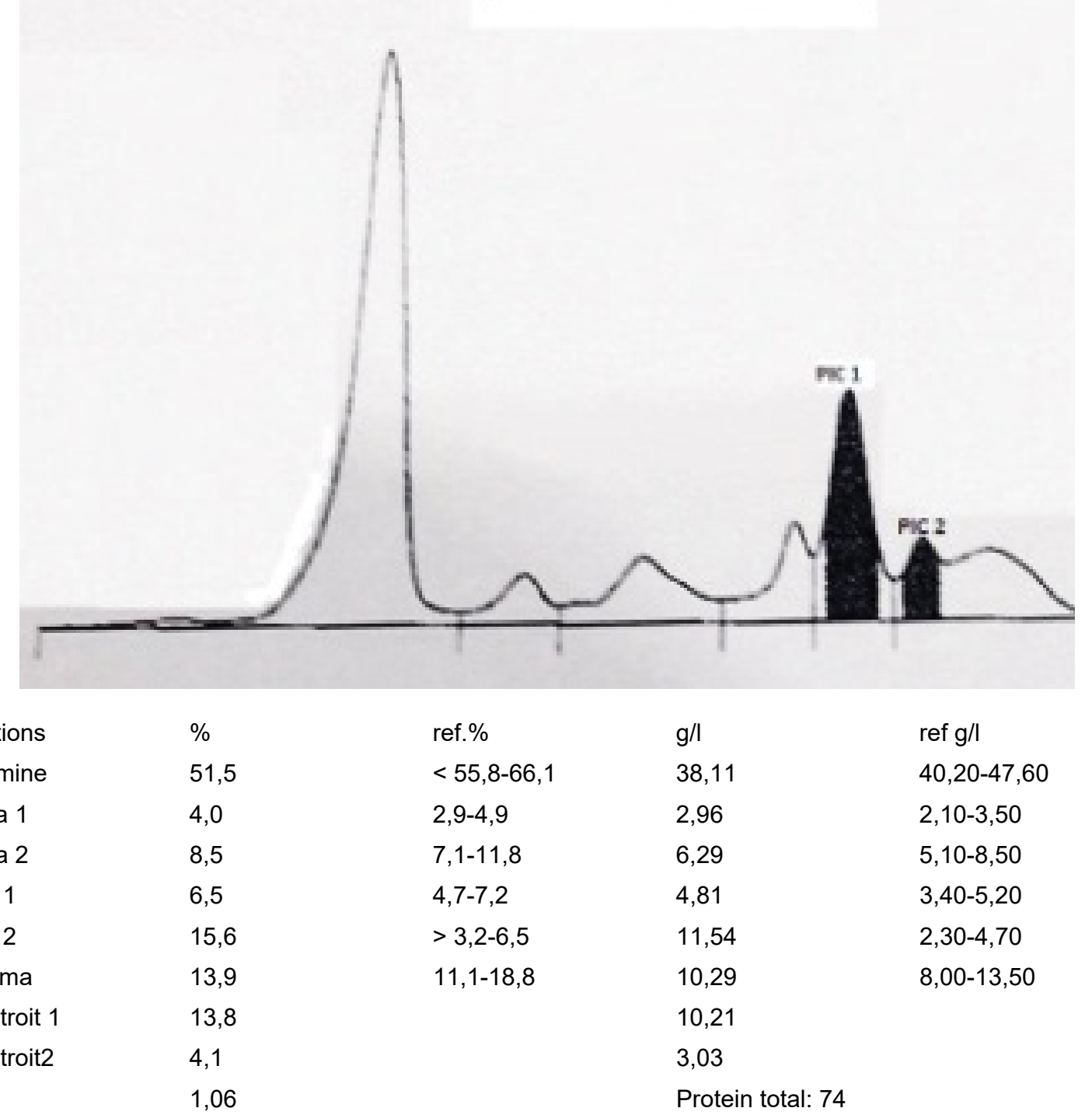

Figure 1: Serum protein electrophoresis shows Band-1 in beta2 region and Band-2 in gamma region. 


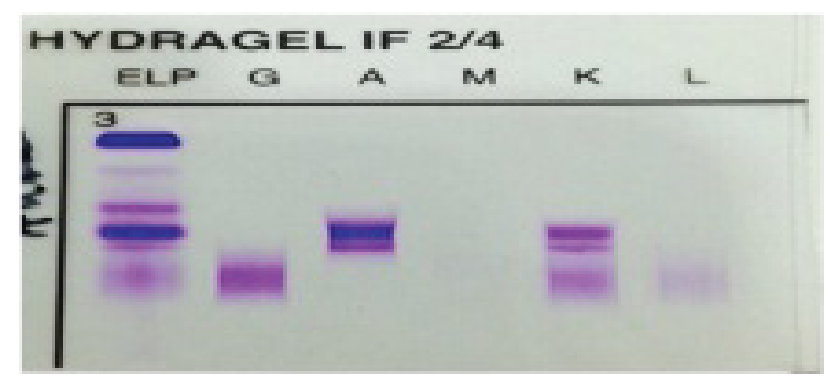

Figure 2: Immunofixation with two bands of IgA-Kappa.

Immunofixation performed on the urine demonstrated a kappa light chain not associated with IgA, IgG, or IgM heavy chains. Renal biopsy is not practiced. Red blood cells morphology showed anisocytosis and no rouloux formation. The bone marrow biopsy demonstrated normocellular marrow with a diffuse interstitial infiltrate of plasma cells comprising more than $30 \%$ of the marrow elements.

Autoimmune serologic test results showed positive tests for antinuclear antibodies (ANA) (title 1:640, homogenous pattern) and antibodies to double stranded DNA (dsDNA) (183AU/ml). Anti-Phospholipid (Antibeta2-Glycoprotein, Anticardiolipin, Antiphosphatidic acid, Antiphosphatidyl inositol and Antiphosphatidyl serine) antibodies were negative (Table 1 ).

SLE was diagnosed according to criteria for SLE set by the American College of Rheumatology (ACR). Based on the above, the simultaneous diagnosis of biclonal gammopathy of IgA kappa variants and systemic lupus erythematosus was made.

\section{Discussion}

Although monoclonal gammopathy is often detected in SLE patients the coexistence of MM has rarely been reported. The prevalence of monoclonal gammopathy of undetermined significance (MGUS) in SLE patients varies from $2.2 \%$ to $3.3 \%$ and the outcome of these patients seems not to differ from the other SLE patients [3].

MM possibly diagnosed roughly a decade after the diagnosis of SLE is made, but it may be diagnosed simultaneously as in our case or even the MM precedes the diagnosis of SLE. A careful literature review confirms that the association of these two diseases has been reported only in a few cases. Pehamberger, Sendagorta, Vaiopoulos and Humayun diagnosed SLE and MM simultaneously [3-6]. To our knowledge, this is the first ever-reported case with biclonal gammopathy of IgA kappa variant and simultaneous SLE.

The pathogenesis of the association between these two disorders is mainly speculative. The genetic factors have been claimed to play a role in the pathogenesis, while some other researchers think that it is the prolonged use of immunosuppressant drugs in previously diagnosed SLE patients which causes MM to develop [3].

Biclonal gammopathies are a vanishingly rare group characterized by the production of two distinct monoclonal proteins. This rare finding can result, for example from either a proliferation of two clones of plasma cells with each producing an unrelated monoclonal spike or from the production of two monoclonal spikes by a single clone of plasma cells. Approximately $1.5 \%$ of $\mathrm{MM}$ cases present with biclonal paraproteinemia [2].

IgA found selectively in the seromucous secretion, tears, saliva and GI secretions. IgA has two subclasses which are differentiated immunochemically as IgA1 and IgA2. Normally the serum contains approximately $90 \%$ of IgA1 molecules [7].

Donald R. Hoffman, Muhammad Younas, Kumar ML and Yadav were reported a case of biclonal gammopathy of IgA-kappa variant [7-10].

\section{Conclusion}

Our case report highlights an extremely rare clinical presentation of biclonal gammpathy of IgA kappa variant and SLE. The association of SLE and MM has rarely been studied so more research needs to be done in this regard so that the definite pathogenetic mechanisms involved can be established and the effective treatment guidelines could be devised.

\section{Conflict of Interest}

None of the authors has any conflict of interest to disclosure.

\section{Authors Contributions}

All the authors have read and agreed to the final manuscript.

\section{References}

1. Bila J, Suvajdzic N, Elezovic I, Colovic M, Boskovic D (2007) Systemic lupus erythematosus and IgA multiple myeloma: A rare association? Med Oncol 24: 445-448.

2. Srinivasan VK, Bhagat P, Bansal F, Chhabra S (2016) Occurrence of double monoclonal bands on protein electrophoresis: An unusual finding. Indian $\mathrm{J}$ Hematol Blood Transfus 32: 184-188.

3. Mohammad H, Iqbal H, Abidullah K, Qasim (2014) SLE and multiple myeloma, a rare and unusual association. $\mathrm{J}$ Med Sci 2: 89-91.

4. Pehamberger H, Diem E, Konrad K (1978) Systemic lupus erythematosus with multiple myeloma. Acta Derm Venereol 58: 527-530.

5. Sendagortata E, Matarredona J, Brieva JA, Rodriguez ML, Ledo A (1987) Systemic lupus erythematosus in association with smoldering multiple myeloma. J Am Acad Dermatol 16: 135-136.

6. Vaiopoulos G, Konstantopoulos K, Mantzourani M, Kaklamanis P (2003) Multiple myeloma associated with systemic lupus erythematosus. Leuk Lymphoma 44: 893894. 
7. Kumar ML, Salma M, Bhulaxmi $P$, Malathi K, Abdullah SK (2014) Biclonal Gammopathy of IgA kappa variant-A case report. IJBR 5: 640-642.

8. Donald RH (2016) An Atlas of Protein Electrophoresis.

9. Muhammad Y, Rizwan H, Farooq (2011) Two monoclonal bands in gamma region on serum protein electrophoresis: A case report. Int J Pathol 9: 29-30.

10. Yadav A, Reddy R, Malathi M (2017) Multiple myeloma with biclonal gammopathy of Iga kappa variant: A case report. Biochem Anal Biochem 6: 345. 